\title{
Heart dose and coronary artery calcification in patients receiving thoracic irradiation for lung cancer
}

\author{
Anel Yakupovich ${ }^{1}$, Mark A. Davison ${ }^{2}$, Michael Z. Kharouta ${ }^{1}$, Julius Turian ${ }^{3}$, Christopher W. Seder $^{4}$, \\ Marta Batus ${ }^{5}$, Louis F. Fogg ${ }^{6}$, Dinesh Kalra ${ }^{1}$, Mark Kosinski ${ }^{1}$, Tuncay Taskesen ${ }^{7}$, Tochukwu M. Okwuosa ${ }^{1}$ \\ ${ }^{1}$ Division of Cardiology, ${ }^{2}$ College of Medicine, ${ }^{3}$ Department of Radiation Oncology, ${ }^{4}$ Department of Cardiovascular and Thoracic Surgery, \\ ${ }^{5}$ Department of Internal Medicine, Rush University Medical Center, Chicago, IL, USA; ${ }^{6}$ Department of Community, Systems and Mental Health \\ Nursing, College of Nursing, Chicago, IL, USA; ${ }^{7}$ Department of Internal Medicine, University of Iowa Health Care Center, Iowa City, Iowa, USA \\ Contributions: (I) Conception and design: T Okwuosa, C Seder, A Yakupovich, J Turian; (II) Administrative support: T Okwuosa; (III) Provision of \\ study materials or patients: A Yakupovich, M Kharouta, M Davison, M Kosinski, D Kalra; (IV) Collection and assembly of data: A Yakupovich, M \\ Kharouta, M Davison, M Kosinski, D Kalra; (V) Data analysis and interpretation: T Okwuosa, C Seder, A Yakupovich, J Turian; (VI) Manuscript \\ writing: All authors; (VII) Final approval of manuscript: All authors. \\ Correspondence to: Tochi M. Okwuosa, DO, FACC, FAHA. Associate Professor of Medicine and Cardiology, Director, Cardio-Oncology Program, \\ Rush University Medical Center, 1717 West Congress Parkway, Kellogg Bldg, Suite 328, Chicago, IL 60612, USA. Email: tokwuosa@rush.edu.
}

Background: Thoracic irradiation (TIR) is associated with an increased risk of coronary artery disease (CAD) and coronary-related death. Lung cancer patients receive considerable doses of TIR, making them a high-risk population that may benefit from post-therapy surveillance. Coronary artery calcium (CAC) is a known biomarker of CAD development and may serve as a useful indicator of disease progression in this population. We hypothesized greater CAC progression in lung cancer patients subjected to higher whole heart radiation doses.

Methods: CAC progression (pre- and >2 years post-TIR) from chest CT scans of lung cancer patients were evaluated. A 2:1 matched control population was established controlling for age, gender, race, and CT scan interval. Vessel-specific CAC presence, progression, and extension in pre- and post-interval CT studies was evaluated by two blinded reviewers using the ordinal method. Dosimetric treatment files were restored and contours of the whole heart and proximal left anterior descending artery (LAD) were created within existing plans to compute radiation doses (Pinnacle Treatment Planning Software). Binary logistic regression analysis identified factors predictive for CAC development. Multiple logistic regression analysis with hierarchal method was used to assess covariates.

Results: Thirty-five patients and 65 controls (50\% female) were evaluated; mean age 57 years, mean follow-up post-radiation $4.9 \pm 2.2$ years. Average mean and maximum left anterior descending coronary artery (LAD) radiation doses were 19.9 Gy (95\% CI, 14.1-25.7) and 30.7 Gy (95\% CI, 23.8-37.5), respectively; 91.6\% inter-observer variability. There was greater incidence of coronary calcification in irradiated patients (48.6\% vs. $24.6 \% ; \mathrm{P}=0.01)$. In interval CT scans, a greater proportion of radiated patients demonstrated new coronary calcification $(\mathrm{P}=0.007)$ and extension within the LAD $(\mathrm{P}=0.003)$. Radiation exposure was the only independent predictor of new calcification (OR 3.1; 95\% CI: 1.09-9.2).

Conclusions: We identified both an increase in the development and progression of CAC in lung cancer patients receiving TIR. Future studies utilizing alternative cancer populations and larger sample sizes are necessary to further correlate radiographic and dosimetric observations to cardiovascular events.

Keywords: Coronary artery calcification (CAC); thoracic irradiation (TIR); lung cancer

Submitted Jun 14, 2019. Accepted for publication Sep 27, 2019.

doi: $10.21037 /$ jtd.2020.01.52

View this article at: http://dx.doi.org/10.21037/jtd.2020.01.52

(c) Journal of Thoracic Disease. All rights reserved. 


\section{Introduction}

Heart disease remains the primary non-malignant cause of death in cancer survivors, with recent evidence suggesting that up to $50 \%$ of cancer patients will develop some degree of cardiovascular complication (1). Thoracic irradiation (TIR) is known to cause microvascular damage and is associated with an increased risk of coronary artery disease (CAD) and coronary related death (2-4). As long-term survival rates and patient lifespan improve, the long-term effects of cancer treatment, specifically cardiotoxicity, are becoming more evident.

Coronary artery calcium (CAC) is associated with coronary plaque accumulation and is a strong predictor of CAD, cardiovascular events, and overall mortality (1-5). CAC scoring has been shown to exceed other generally accepted screening methodologies for assessing cardiovascular disease risk (6). Alluri et al. demonstrated improved health outcomes in patients that received CAC imaging as a CAD screening modality during times of lung cancer surveillance, typically in patients with concerns for recurrence (4).

Despite improvements in modern radiotherapy techniques, cancer patients receiving TIR still sustain considerable doses to surrounding organs, including the heart (7). A study of 2,168 female patients that received radiotherapy for breast cancer demonstrated that the rate of major coronary events was directly related to mean radiation dose to the heart. Each additional gray (Gy) to the heart was associated with a $7.4 \%$ increase in coronary events (95\% CI, 2.9-14.5, $\mathrm{P}<0.001$ ) (7). Additionally, the anterior location of the left anterior descending (LAD) coronary artery suggests that radiation to the LAD may also serve as an independent predictor of cardiovascular events (8).

The goal of this study is to characterize the presence, severity and progression of CAC in cancer patients post TIR. We hypothesized that CAC presence, severity and progression is increased in lung cancer patients who received high levels of TIR in comparison to those that had received less radiation or no radiation at all.

\section{Methods}

\section{Study design}

We conducted a retrospective cohort study with an approximate 2:1 matched control population. All protocols were pre-approved by the institutional review board at Rush University Medical Center (RUMC). Patient group was identified from the RUMC cancer registry and included patients diagnosed with lung cancer between 2007 and 2012, age $18-80$ years old; and whom received TIR. We excluded patients that did not receive follow-up care at RUMC, those without serial chest computed tomography (CT) surveillance at RUMC following their lung cancer treatment, patients who died within 2-years of cancer diagnosis.

A 2:1 matched control population was created consisting of lung cancer patients that did not receive therapeutic TIR. Matched parameters included age, gender, race, and comparable CT study time intervals. Hospital records of all the patients were retrospectively reviewed for clinical characteristics and comorbidities including obesity (BMI $>30$ $\mathrm{kg} / \mathrm{m}^{2}$ ), hypertension, diabetes mellitus, dyslipidemia, and smoking history (9). Additionally, a medication review was conducted, specifically examining for the use of angiotensin converting enzyme inhibitors (ACE-I), beta-blockers, nitrates, aspirin and statins at the time of their first CT date.

\section{CAC quantification}

Chest CT scans were obtained using the GE BrightSpeed CT system. Axial acquisitions were obtained through the chest, and multiple reformatted images were generated; including coronal and sagittal multi-planar reformation (MPR) images; coronal and axial maximum intensity projection (MIP) images. The CT scans were performed with attention to patient radiation dose reduction, as low as reasonably achievable (ALARA), while maintaining diagnostic image quality. At least one of the following dose reduction techniques was used: automated exposure control, adjustment of the $\mathrm{mA}$ and/or $\mathrm{kV}$ according to patient size, use of iterative reconstruction technique. Radiation Shields were used.

High-resolution CT images obtained prior to cancer diagnosis were utilized for baseline CAC measurements. Each patient received 2 CT scans. Post-treatment chest CT studies following TIR were utilized for computation of CAC progression metrics throughout the imaging interval. These post-treatment CT scans were as far apart as possible from at least 2 years, to a maximum time of 7 years apart. CT images for control patients were selected within one year of their study patient counterpart's pre- and postinterval dates.

Chest CT images were assessed for presence, severity and extension of CAC by 2 independent physician reviewers, whom were blinded to the patient's history and cohort assignment. Presence of CAC was viewed as any 
visualization of calcium within the cardiovascular structures listed below. Severity was viewed as an increase of calcium within a single focus, while extension was viewed as an increase of calcium within more foci. The cardiovascular structures examined within the CT images included the left main (LM), Left anterior descending, left circumflex (Cx), right (RCA) coronary arteries; aortic arch and descending aorta.

Circle Cardiovascular Imaging and Centricity GE software was used to assess the CAC data. CAC presence or absence was defined as a categorical value of Yes $=1$, No $=0$. Each vessel was given a score of 0 to 3 for extent defined as $0=$ no foci; 1 = focal, a single focus; 2 = moderate, $>1$ focus; or diffuse = 3 , foci in proximal, mid-, and distal segments. Each vessel was also given a score of 0 to 3 for severity defined as $0=$ no foci, 1 = mild, 2 = moderate and 3 = severe. The Scale for extension of CAC; None: No changes; Mild: One-degree changes (none to mild; mild to moderate, moderate to severe); Moderate: 2-degree changes (none to moderate, mild to severe); Severe: 3-degree changes (none to severe) (9).

\section{Radiation dosimetry}

Treatment planning records from the patient group were retrieved from MOSAIQ the electronic radiation oncology records database. Dosimetric treatment records were dearchived and restored in the treatment planning system (TPS), Pinnacle (Phillips; Amsterdam, Netherlands). Whole heart contours were created within existing plans and the TIR doses were computed. Heart contours began one slice above the aortic valve, at the approximate level of the ostia, and continued inferiorly to the cardiac apex.

Similarly, proximal LAD contours were generated to compute the respective radiation doses. Specifically, contours were determined by most recent, pre-treatment chest CT scan. Pinnacle CT-to-CT fusion software was used to fuse these images with the simulation CT using a mutual information-based algorithm. The heart/LAD volumes, maximum doses, mean doses and $\mathrm{V}_{5}-\mathrm{V}_{70}$ were determined based on the original plan.

\section{Statistical analysis}

Descriptive statistics were calculated for the study variables. The Student's t-test was used to compare quantitative variables between patient group and controls, and continuous data were presented as mean $\pm \mathrm{SD}$. The Chi square test or Fisher's exact test was used to compare qualitative variables, and categorical variables were summarized as percentages.
Binary logistic regression analysis was used to identify factors associated with and predictive for development of CAC. Multiple logistic regression analysis with hierarchal method was used to assess the predictive effect of variables like smoking, radiation exposure, dyslipidemia, and duration between CT on CAC development after correction for baseline confounding factors. Interobserver variability coefficient was computed for both independent CAC reviewers. SPSS IBM 23 was used for all statistical analysis and value of $<0.05$ was considered statistically significant.

\section{Results}

We identified a total of 35 patients based on set inclusion and exclusion criteria, matched with 65 controls in an approximately $2: 1$ ratio. The number of participants in the control group were limited in the study and 5 patients were unable to be matched in a 2:1 fashion due to a lack of similar CT time interval. The average mean and maximum radiation doses to the whole heart were 11.61 Gy $(95 \%$ CI: 8.0-15.2) and 52.1 Gy (95\% CI: 41.8-55.9); while the average mean and maximum doses to the proximal LAD were 19.9 Gy (95\% CI: 14.1-25.7) and 30.7 Gy (95\% CI: 23.8-37.5) respectively. Baseline characteristics including use of known cardio-protective medications for patients and controls are shown in Table 1. The mean age for the study population was 57 years. There were significantly more smokers $(\mathrm{P}<0.001)$ and lower $\mathrm{BMI}$ in the treatment compared with the control group $(26 \pm 6.9$ vs. $29 \pm 8.2$; $\mathrm{P}=0.03)$. The control group was comprised of more patients utilizing aspirin $(\mathrm{P}=0.01)$ and $\mathrm{ACE}-\mathrm{I}(\mathrm{P}<0.001)$.

The presence, extent, and severity of CAC progression in the patient group (post TIR), and controls, are included in Tables 2, 3 and 4, respectively. A higher number of the patient group developed CAC in one or more major coronary arteries compared with controls (48.6\% vs. $24.6 \%$; $\mathrm{P}=0.01$ ) (Table 2). In particular, CAC development was significantly higher in the $\operatorname{LAD}(\mathrm{P}=0.007)$ and $\mathrm{Cx}(\mathrm{P}=0.01)$. Additionally, there was significant $\mathrm{CAC}$ extension in the patient group for LAD ( $\mathrm{P}=0.003), \mathrm{Cx}(\mathrm{P}=0.03)$, aortic arch $(\mathrm{P}=0.003)$ and descending aorta $(\mathrm{P}=0.009)$, compared with controls (Table 3). Similarly, progression in severity of CAC was significantly increased in the LAD $(\mathrm{P}=0.005)$ and $\mathrm{Cx}$ $(\mathrm{P}=0.01)$ of the patient group, compared with the control group (Table 4). The calculated inter-observer reliability between both CT readers was $91.6 \%$.

Unadjusted logistic regression was used to analyze the characteristics of our study population with newly developed 
Table 1 Baseline characteristics of study population

\begin{tabular}{|c|c|c|c|}
\hline Baseline characteristics & Patient group $(\mathrm{n}=35)$ & Control group $(n=65)$ & $P$ value \\
\hline Gender (male), n (\%) & $18(51.4)$ & $32(49.2)$ & 0.8 \\
\hline BMI & $26 \pm 6.9$ & $29 \pm 8.2$ & 0.03 \\
\hline Obesity, n (\%) & $12(34.3)$ & $28(43.1)$ & 0.3 \\
\hline Diabetes mellitus, n (\%) & $7(20.0)$ & $17(26.2)$ & 0.6 \\
\hline Dyslipidemia, n (\%) & $14(40.0)$ & $39(60.0)$ & 0.056 \\
\hline Smoker, n (\%) & $35(100.0)$ & $41(63.0)$ & $<0.001$ \\
\hline Time between CTs (years) & $4.9 \pm 2.2$ & $4.7 \pm 2.1$ & 0.7 \\
\hline Nitrate, n (\%) & $2(5.7)$ & $3(4.6)$ & 0.8 \\
\hline ASA, $n(\%)$ & $9(25.7)$ & $34(52.3)$ & 0.01 \\
\hline Statin, n (\%) & $21(60.0)$ & $36(55.4)$ & 0.6 \\
\hline Ethnicity, n (\%) & & & 0.4 \\
\hline White & $22(62.9)$ & $39(60.0)$ & \\
\hline African American & $13(37.1)$ & $23(35.4)$ & \\
\hline Hispanic & $0(0)$ & $3(4.6)$ & \\
\hline
\end{tabular}

Data are presented as the mean value $\pm S D$ ( $P$ values for independent student t-test) or number of patients (percentage) $(P$ values for chisquare test). ACE-I, angiotensin converting enzyme inhibitor; ASA, acetyl salicylic acid; CT, computer tomography; BMI, body mass index.

CAC in at least one coronary artery during follow up (Table 5); and showed that radiation exposure $(\mathrm{P}=0.01)$ and longer time duration between $\mathrm{CT}$ scans $(\mathrm{P}=0.02)$ are two independent variables that significantly correlated with development of new CAC. With an adjusted model (Table 6), radiation exposure was the only independent variable that significantly predicted new CAC development (OR 3.1; 95\% CI: 1.09-9.2).

\section{Discussion}

While CAC based models have been shown to predict future cardiovascular events and mortality beyond the traditional Framingham risk criteria (6), the use of CAC in assessing cardiac risk in cancer patients having undergone cancer therapy remains largely unknown. In our study, we identified both an increase in the presence and progression of both severity and extent of CAC in lung cancer patients receiving TIR. Furthermore, our study showed that after multivariable adjustments, radiation exposure was the only independent variable that significantly predicted new CAC development. A strength of our study is that we evaluated patients with lung cancer (whom usually receive higher doses of TIR), and identified increased development, progression, and severity of CAC in these patients, compared with non-irradiated controls. Our study, unlike others, did not compare baseline CAC data to published values for CAC, but instead utilized a rigorously matched control population without any reported TIR. This not only increased the power of the study, but also allowed us to include a common population with similar exposures. To the best of our knowledge, this is the first study to examine the development, extension and progression of CAC in lung cancer patients exposed to TIR. This data suggests that despite improvements in TIR techniques, lung cancer patients still receive a radiation dose that may lead to the development of CAC in as little as 2 years after treatment. We particularly utilized lung cancer patients for our study 
Table 2 Presence of CAC after TIR

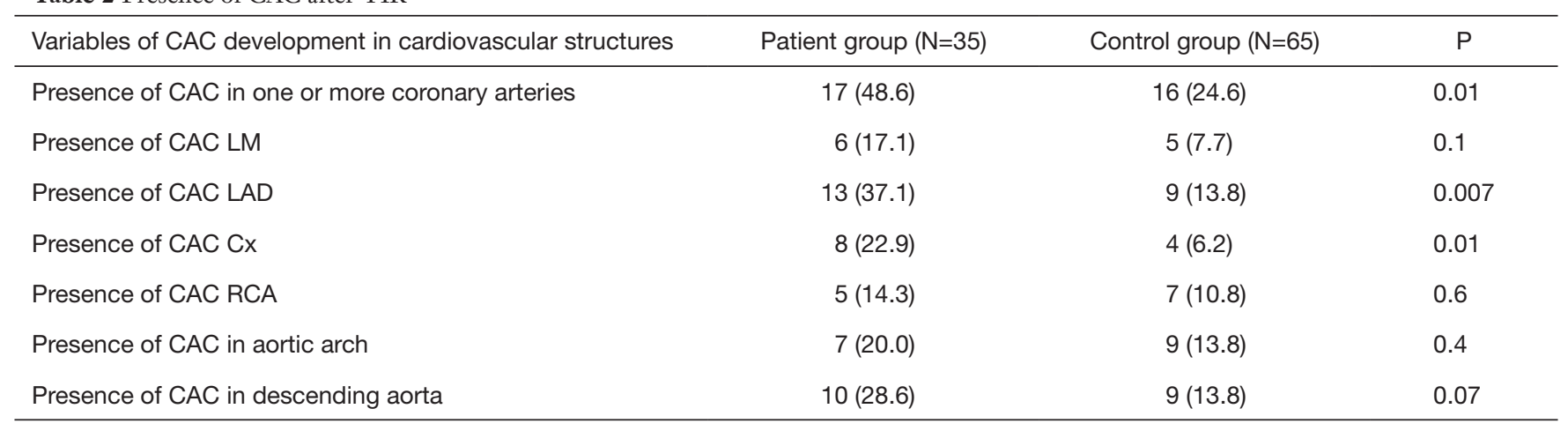

Table demonstrates CAC presence after TIR in patient group and baseline presence of CAC developed in matched CT interval times in control group that did not receive TIR. Data showed in number (percentage) for patients with new developed CAC (P values for chisquare test). CAC, coronary artery calcification; TIR, thoracic irradiation; Cx, left circumflex coronary artery; LAD, left anterior descending coronary artery; LM, left main coronary artery; RCA, right coronary artery.

Table 3 Extension of CAC after TIR

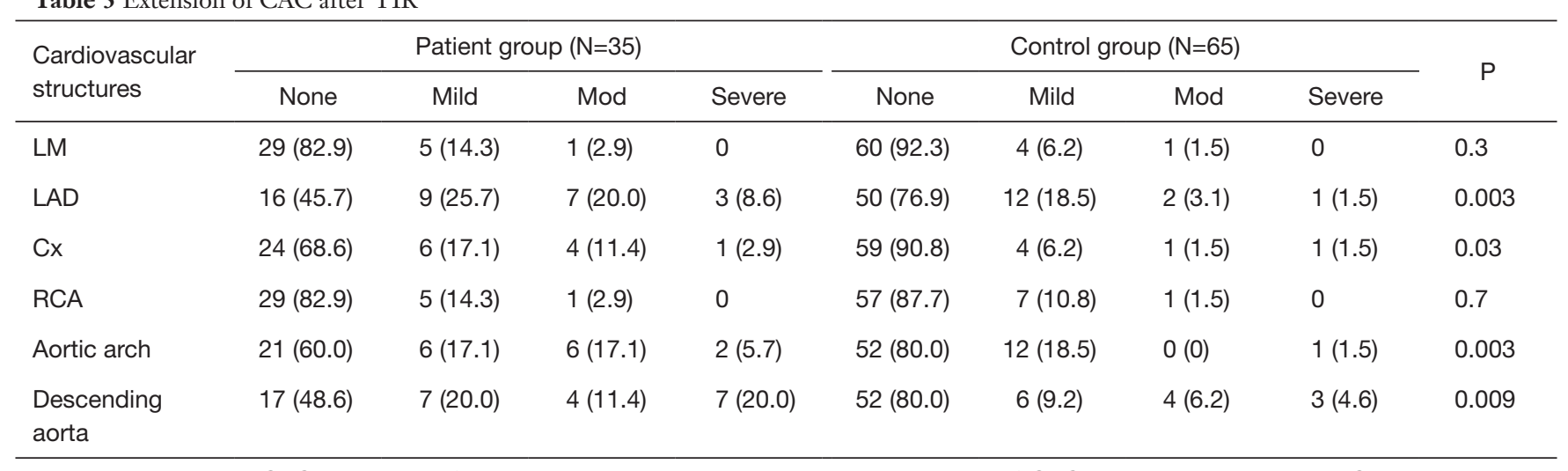

Table demonstrates CAC extension after TIR in patient group and baseline extension of CAC developed in matched CT interval times in control group that did not receive TIR. Data showed in number (percentage) for patients with progression of CAC extension (P values for chi-square test). Scale for extension of CAC: None, No changes; Mild, 1 degree changes (none to mild; mild to moderate, moderate to severe); Moderate: 2 degree changes (none to moderate, mild to severe); Severe: 3 degree changes (none to severe). CAC, coronary artery calcification; TIR, thoracic irradiation; Cx, left circumflex coronary artery; LAD, left anterior descending coronary artery; LM, left main coronary artery; RCA, right coronary artery.

because of the higher radiation dose to the chest and heart, which would allow us observe results in a small number of patients within a shorter period of time.

Micro- and macro-vascular injury in tissues receiving radiotherapy is considered the main cause of radiation-induced heart disease (7). Radiotherapy causes acute endothelial inflammation leading to the mobilization of granulocytes, monocytes, and macrophages that may lead to fibrosis and marked thickening of the adventitia and media of the arteries (8). Eventually this leads to stenosis and calcifications and accelerates progression to unstable atherosclerosis.

We aimed to measure this atherosclerosis by utilizing a visually assessed CAC score. As previously mentioned, calculated CAC scores have been shown to exceed other generally accepted screening methodologies for assessing cardiovascular disease risk $(10,11)$. In comparison to the general population, cancer survivors experience an increase in the risk of coronary arterial atheroscleroticrelated events including $\mathrm{CAD}$, angina, and myocardial infarction (12). This begged the question of whether CAC would also develop in cancer patients as a result of TIR; thereby forming a useful screening tool for this population. Whitlock et al. demonstrated in the large multi-ethnic atherosclerosis (MESA) study, that the development of CAC occurs in many types of cancer patients even after accounting for other atherosclerotic risk factors (13). 
Table 4 Progression of CAC severity after TIR

\begin{tabular}{|c|c|c|c|c|c|c|c|c|c|}
\hline $\begin{array}{l}\text { Cardiovascular } \\
\text { structures }\end{array}$ & \multicolumn{4}{|c|}{ Patient group ( $\mathrm{N}=35)$} & \multicolumn{4}{|c|}{ Control group ( $\mathrm{N}=65)$} & $\mathrm{P}$ \\
\hline LM & $28(80.0)$ & $5(14.3)$ & 0 & $2(5.7)$ & $59(90.8)$ & $4(6.2)$ & $2(3.1)$ & 0 & 0.08 \\
\hline LAD & $16(45.7)$ & $11(31.4)$ & $6(17.1)$ & $2(5.7)$ & $48(73.8)$ & $15(23.1)$ & $2(3.1)$ & 0 & 0.005 \\
\hline$C x$ & $23(65.7)$ & $11(31.4)$ & 0 & $1(2.9)$ & $57(87.7)$ & $6(9.2)$ & $2(3.1)$ & 0 & 0.01 \\
\hline Aortic arch & $20(57.1)$ & $10(28.6)$ & $5(14.3)$ & 0 & $49(75.4)$ & $13(20.0)$ & $3(4.6)$ & 0 & 0.1 \\
\hline $\begin{array}{l}\text { Descending } \\
\text { aorta }\end{array}$ & $15(42.9)$ & $12(34.3)$ & $5(14.3)$ & $3(8.6)$ & $43(58.0)$ & $15(23.1)$ & $6(9.2)$ & $1(1.5)$ & 0.08 \\
\hline
\end{tabular}

Table demonstrates CAC progression after TIR in patient group and baseline progression of CAC developed in matched CT interval times in control group that did not receive TIR. Data showed in number (percentage) for patients with progression of CAC severity ( $\mathrm{P}$ values for chi-square test). Scale for extension of CAC; None: No changes; Mild: One-degree changes (none to mild; mild to moderate, moderate to severe); Moderate: Two-degree changes (none to moderate, mild to severe); Severe: Three-degree changes (none to severe). CAC, coronary artery calcification; TIR, thoracic irradiation; Cx, left circumflex coronary artery; LAD, left anterior descending coronary artery; LM, left main coronary artery; RCA, right coronary artery.

Table 5 Binary logistic regression analysis to predict new CAC development

\begin{tabular}{llccc}
\hline Variables & B & P value & OR (EXP B) & 95\% confidence interval \\
\hline Time between CTs (years) & 0.23 & 0.02 & 1.2 & $1.03-1.5$ \\
Radiation exposure (Gy) & 1.06 & 0.01 & 2.8 & $1.2-6.9$ \\
Smoking & 1.1 & 0.059 & 3.08 & $0.95-9.9$ \\
Age & -0.02 & 0.3 & 0.9 & $0.91-1.03$ \\
BMI & 0.009 & 0.7 & 1 & $0.95-1.06$ \\
Dyslipidemia & 0.8 & 0.057 & 2.3 & $0.97-5.5$ \\
DM & 0.49 & 0.3 & 1.6 & $0.6-4.2$ \\
HTN & 0.09 & 0.8 & 0.9 & $0.3-2.3$ \\
Statin use & 0.8 & 0.07 & 2.2 & $0.9-5.4$ \\
ACEl use & 0.86 & 0.05 & 2.3 & $1-5.6$ \\
\hline
\end{tabular}

CAC, coronary artery calcification; CT, computed tomography; BMI, body mass index; Gy, Gray; DM, diabetes mellitus; HTN, hypertension; $\mathrm{ACEl}$, angiotensin converting enzyme inhibitor.

However, they were unable to show a significant progression of CAC in individuals they found with pre-existing CAC. This led them to question the potential mechanisms for the development of CAC in cancer survivors. In particular, their analysis indicated that the progression of atherosclerosis that can be detected on imaging. It is noteworthy that their study evaluated all comers with cancer who received various treatments. Conversely, our study focused on patients particularly post (high dose) TIR.

Several other studies have investigated the potential impact of cancer and its therapies on CAC. In a cohort of breast cancer patients prior to chemotherapy or radiation, Mast et al. demonstrated increased CAC in middle aged women as compared to age-matched controls (14). Another case series of 47 Hodgkin's lymphoma patients treated with radiation found abnormally high CAC scores as compared to the published values for CAC (15). A similar earlier series of Hodgkin's lymphoma patients at a median age of 45 years treated with mediastinal radiotherapy approximately 26 years earlier were also reported to have CAC above the $90^{\text {th }}$ 
Table 6 Hierarchical logistic regression analysis to predict new CAC development

\begin{tabular}{lcccr}
\hline Variables & B & P value & OR (EXP B) & 95\% confidence interval \\
\hline Time line between CT's & 0.15 & 0.17 & 1.1 & $0.9-1.4$ \\
Radiation exposure & 1.15 & 0.03 & 3.1 & $1.09-9.2$ \\
Smoking & 0.58 & 0.4 & 1.7 & $0.45-7.05$ \\
Dyslipidemia & 0.8 & 0.057 & 2.3 & $0.97-5.5$ \\
\hline
\end{tabular}

CAC, coronary artery calcification; CT, computed tomography.

percentile for age and gender reference values (16). Despite this evidence supporting the development of CAC at various stages of diagnosis or remission, other studies suggest no changes in CAC. For example, a recent cohort of 236 breast cancer survivors who had undergone radiation and/ or chemotherapy compared CAC with published general population CAC scores, and showed a similar age-matched distribution (10). Our study is unique as described below.

Unlike these published literatures that have matched patients with historical controls, our study compared a patient group with a carefully selected population of controls, and likely explains our significant results. In our study, both men and women with lung cancer diagnosed between 2007 to 2013 were included if they had survived both diagnoses and treatment for at least 24 months. The reason for this timeline is due to the perceived effects of TIR on the heart. Most evidence estimated aggregate incidence of radiotherapy-induced ischemic heart disease to be $10-30 \%$ by $5-10$ years post treatment (2). However, a population-based case control study of breast cancer patients by Darby et al. outlined that increased exposure of the heart to ionizing radiation during TIR increased the rate of subsequent ischemic heart disease proportional to the mean dose received by the heart, and can begin within 2 years after exposure and continue for at least 20 years (7). Furthermore, the risk of major coronary events increased linearly with mean dose of radiation to the heart, with the magnitude of the risk increasing by $7.4 \%$ per gray $(7,17)$. It should be noted that TIR regimens have changed since the women in these trials were irradiated and the doses of radiation to which the heart is exposed are now generally lower. During Darby's study, the mean dose to the whole heart was 4.9 Gy (range, 0.03-27.72 Gy), compared with $11.61 \mathrm{~Gy}$ in our study. These results support Darby's study by showing that higher radiation doses continue to contribute to an increased risk of CAD. Our study utilized the development and progression of $\mathrm{CAC}$ as a surrogate for future $\mathrm{CAD}$.

On average, lung cancer patients receiving radiotherapy treatment since 2007 unintentionally receive a 4.1 Gy dose to the heart (18). Considering the high doses of TIR administered to lung cancer patients, this population lends itself to the study of CAC, a biomarker of CAD and predictor of major coronary events (19). Additionally, patients with lung cancer require lifelong surveillance for recurrence at intervals ranging from 3-12 months. Although the images acquired are considered low dose/quality, Dirriches et al. in 2015 was able to adequately evaluate CAC (20). The value of CAC seen in routine screening/ surveillance CT scan to predict clinical outcomes was also seen in 3,559 male current or former smokers who underwent a CT chest scan as part a lung cancer screening program $(9,21)$. CAC was associated with increased risk of both fatal and non-fatal cardiovascular events over a median follow up of 2.9 years. The detection of extensive CAC (Agatston $>400$ ) in this population was associated with an increased hazard ratio of 12.58 (95\% CI: 5.42-29.16) in comparison to patients with no coronary calcification $(17,20)$.

The proximal LAD was of particular interest due to the anatomical localization and reference in previous investigations $(21,22)$. According to Darby et al., the mean heart dose was a better predictor of the rate of major coronary events than the mean dose to the LAD (7). However, despite the historical changes in radiotherapy techniques and heart doses, the LAD lying on the anterior myocardial wall still received an average dose of 20 Gy during breast cancer treatment in the 1980s and has been linked to a risk of ischemic heart disease for years after radiotherapy (8). In our study, the average mean and maximum doses to the proximal LAD were approximately 19.86 and $30.68 \mathrm{~Gy}$, respectively. These results may indicate relevant late cardiac toxicity and may support routine delineation of the vessel during TIR planning to further avoid non-targeted radiotherapy; particularly because lung cancer patients are living longer with improved targeted cancer therapies.

Lung cancer patients are ideal for detecting CAC due to 
recommended surveillance for recurrence. Moreover, CAC detection is relatively safe and inexpensive when compared to other common CAD detecting techniques such as a stress echocardiography, coronary CT angiography or stress myocardial perfusion studies $(17,19)$. Specifically, these alternative detection modalities pose a greater risk to patients; often involving higher radiation dose (which may increase the risk of subsequent malignancy), and intravenous contrast (which can induce nephropathy and renal damage) (23). In this study, we showed that we could possibly substitute other more-involved, time-consuming, more expensive detection modalities for simple CAC screening on the already necessary surveillance chest CT scans in lung (and likely other) cancer survivors (24). Of note, $74.3 \%$ (26 patients) of the patients undergoing TIR quit smoking by the conclusion of their radiation treatment course.

Our study was limited by the small size of the cohort $(n=35)$, made stronger by a meticulously matched selection of 2:1 controls to increase the power of the study. Additionally, this is a single-center study limiting the variability of the population type and potential external validity. In order to elucidate the effect of TIR, strong exclusion criteria were included such as those who did not have post CT images at least 2 years after initial treatment. This may have introduced selection bias; but if anything, should have diluted our study findings. Furthermore, the current study only included lung cancer patients rather than other cancer patients receiving TIR. Despite these limitations, the adjusted logistic regression analysis did not suggest that there were significant differences between patients and controls in our study. Although the control group did have more dyslipidemia (59.6\% vs. $40 \%)$ and were receiving more cardiovascular medications including aspirin, ACE-I and beta blocker, these variables were adjusted for in our regression analysis, and TIR still independently predicated new development of CAC. The retrospective nature of our study meant that we were unable to determine a true baseline CAC particularly in the control population; however, our patient and control groups were matched based on time intervals between CT scans. Further study limitations include the use of ordinal visualized CAC scoring instead of gold standard Agatston score. This was necessary to prove our case that routine screening/surveillance chest CT scans could be utilized for CAC assessment in patients post TIR. In addition, prior studies have demonstrated that a simple, visually assessed CAC score was strongly correlated with the Agatston score and had similar predictive values for CAD death (24).

\section{Conclusions}

We found increased and newly developed CAC in lung cancer patients exposed to TIR compared with matched controls who were not irradiated. In multiple logistic regression analysis, the strongest independent predictor of new development of CAC was TIR. This study identifies CT-based CAC screening as a potential modality for $\mathrm{CAD}$ screening in cancer patients following TIR. Larger trials linking these study findings to major cardiovascular events are required.

\section{Acknowledgments}

Funding: None.

\section{Footnote}

Conflicts of Interest: CWS serves as the unpaid editorial board member of Fournal of Thoracic Disease from Jul 2019 to Jun 2021. The other authors have no conflicts of interest to declare.

Ethical Statement: The authors are accountable for all aspects of the work in ensuring that questions related to the accuracy or integrity of any part of the work are appropriately investigated and resolved. All protocols were pre-approved by the institutional review board at Rush University Medical Center (No. 15041514).

Open Access Statement: This is an Open Access article distributed in accordance with the Creative Commons Attribution-NonCommercial-NoDerivs 4.0 International License (CC BY-NC-ND 4.0), which permits the noncommercial replication and distribution of the article with the strict proviso that no changes or edits are made and the original work is properly cited (including links to both the formal publication through the relevant DOI and the license). See: https://creativecommons.org/licenses/by-nc-nd/4.0/.

\section{References}

1. Chen CL. Cardiovascular prevention in the cancer survivor. Curr Atheroscler Rep 2015;17:484.

2. Lancellotti P, Nkomo VT, Badano LP, et al. Expert consensus for multi-modality imaging evaluation of cardiovascular complications of radiotherapy in adults: a report from the European Association of Cardiovascular Imaging and the American Society of Echocardiography. J 
Am Soc Echocardiogr 2013;26:1013-32.

3. Lipshultz SE, Adams MJ, Colan SD, et al. Long-term cardiovascular toxicity in children, adolescents, and young adults who receive cancer therapy: pathophysiology, course, monitoring, management, prevention, and research directions: a scientific statement from the American heart association. Circulation 2013;128:1927-95.

4. Alluri K, Joshi P H, Henry T S, et al. Scoring of coronary artery calcium scans: History, assumptions, current limitations, and future directions. Atherosclerosis 2015;239:109-17.

5. Clarke M, Collins R, Darby S, et al. Effects of radiotherapy and of differences in the extent of surgery for early breast cancer on local recurrence and 15-year survival: an overview of the randomized trials. Lancet 2005;366:2087-106.

6. Hughes-Austin JM, Dominguez A, Allison MA, et al. Relationship of coronary calcium on standard chest CT scans with mortality. JACC Cardiovasc Imaging 2016;9:152-9.

7. Darby SC, Ewertz M, McGale P, et al. Risk of ischemic heart disease in women after radiotherapy for breast cancer. N Engl J Med 2013;368:987-98.

8. Sinacki M, Serkies K, Dziadziuszko R, et al. Radiation dose to the left anterior descending coronary artery during interstitial pulsed-dose-rate brachytherapy used as a boost in breast cancer patients undergoing organ-sparing treatment. J Contemp Brachytherapy 2017;9:7-13.

9. Edwards BK, Noone AM, Mariotto AB, et al. Annual Report to the Nation on the status of cancer, 1975-2010, Featuring prevalence of comorbidity and impact on survival among persons with lung, colorectal, breast, or prostate cancer. Cancer 2014;120:1290-314.

10. Tjessem KH, Bosse G, Fossa K, et al. Coronary calcium score in 12-year breast cancer survivors after adjuvant radiotherapy with low to moderate heart exposurerelationship to cardiac radiation dose and cardiovascular risk factors. Radiother Oncol 2015;114:328-34.

11. Williams KA Sr, Kim JT, Holohan KM. Frequency of unrecognized, unreported, or underreported coronary artery and cardiovascular calcification on noncardiac chest CT. J Cardiovasc Comput Tomogr 2013;7:167-72.

12. Hooning MJ, Botma A, Aleman BMP, et al. Long-term risk of cardiovascular disease in 10-year survivors of breast cancer. J Natl Cancer Inst 2007;99:365-75.

13. Whitlock MC, Yeboah J, Burke GL, et al. Cancer and Its Association with the Development of Coronary Artery Calcification: An Assessment From the Multi-Ethnic Study of Atherosclerosis. J Am Heart Assoc 2015;4. doi: 10.1161/ JAHA.115.002533.

14. Mast ME, Heijenbrok MW, Petoukhova AL, et al. Preradiotherapy calcium scores of the coronary arteries in a cohort of women with early-stage breast cancer: a comparison with a cohort of healthy women. Int J Radiat Oncol Biol Phys 2012;83:853-8.

15. Roychoudhuri R, Robinson D, Putcha V, et al. Increased cardiovascular mortality more than fifteen years after radiotherapy for breast cancer: a population-based study. BMC Cancer 2007;7:9.

16. Andersen R, Wethal T, G€unther A, et al. Relation of coronary artery calcium score to premature coronary artery disease in survivors $>15$ years of Hodgkin's lymphoma. Am J Cardiol 2010;105:149-52.

17. Liu C, Gong G, Guo C, Liu T, et al. The feasibility of evaluating radiation dose to the heart by integrating kilovoltage-cone beam computed tomography in stereotactic body radiotherapy of early non-small-cell lung cancer patients. Radiat Oncol 2013;8:295.

18. Ming X, Feng Y, Yang C, et al. Radiation-induced heart disease in lung cancer radiotherapy: A dosimetric update. Medicine (Baltimore) 2016;95:e5051.

19. Polonsky TS, McClelland RL, Jorgensen NW, et al. Coronary artery calcium score and risk classification for coronary heart disease prediction. JAMA 2010;303:1610-6.

20. Dirrichs T, Penzkofer T, Reinartz SD, et al. Extracoronary thoracic and coronary artery calcifications on chest CT for lung cancer screening: association with established cardiovascular risk factors - the "CT-risk" trial. Acad Radiol 2015;22:880-9.

21. Feng M, Moran JM, Koelling T, et al. Development and validation of a heart atlas to study cardiac exposure to radiation following treatment for breast cancer. Int J Radiat Oncol Biol Phys 2011;79:10-8.

22. Gagliardi G, Constine LS, Moiseenko V, et al. Radiation dose-volume effects in the heart. Int J Radiat Oncol Biol Phys 2010;76:S77-85.

23. Yeboa DN, Evans SB. Contemporary breast radiotherapy and cardiac toxicity. Semin Radiat Oncol 2016;26:71-8.

24. Greenland P, Bonow RO, Brundage BH, et al. ACCF/ AHA 2007 clinical expert consensus document on coronary artery calcium scoring by computed tomography in global cardiovascular risk assessment and in evaluation of patients with chest pains: a report of the American College of Cardiology Foundation Clinical Expert Consensus task force. J Am Coll Cardiol 2007;49:378-402.

Cite this article as: Yakupovich A, Davison MA, Kharouta MZ, Turian J, Seder CW, Batus M, Fogg LF, Kalra D, Kosinski M, Taskesen T, Okwuosa TM. Heart dose and coronary artery calcification in patients receiving thoracic irradiation for lung cancer. J Thorac Dis 2020;12(3):223-231. doi: 10.21037/jtd.2020.01.52 